\section{Do not inflate exponentially the evidence for the polynomial model: A reply to Jones}

\author{
GIDEON KEREN \\ Institute for Perception TNO \\ Soesterberg, The Netherlands
}

A well-documented phenomenon supported by several studies is that exponential growth functions are grossly underestimated by human subjects (Keren, 1983; Wagenaar \& Sagaria, 1975; Wagenaar \& Timmers, 1978, 1979). What is less clear is the nature of the computational processes used by subjects that lead to underestimation. Keren (1983), following Wagenaar and his collaborators, adopted a model that assumes that subjects correctly perceive such series to be exponentially distributed but incorrectly perceive their parameters. Jones (1984), reiterating previous claims (Jones, 1977, 1979), proposed a polynomial model which, according to his claim, fits the data best. The main point of Keren's (1983) article was to show that cultural differences or, more accurately, differences of everyday experience with series that are approximately exponentially distributed (i.e., rate of inflation) may affect subjects' perceptions and extrapolations of future values. Jones (1984), using his polynomial model and applying it to Keren's data, has reached the same conclusion. Hence, both the polynomial and the exponential model lead to the same conclusion proposed by Keren. Nevertheless, it is still of interest to find out which is the correct model. In other words, for both theoretical as well as applied purposes, it is important to find out the underlying computational algorithms that are used by subjects.

Unfortunately, the experimental data reported by Keren (1983) and by Wagenaar and his collaborators cannot provide a strict test to determine which model is more appropriate. To investigate the computational mechanisms that are used by subjects to make their estimates, further (and different) experiments are needed. Jones's claim that the polynomial model is a viable one and that the exponential model is not is, however, unfounded, as shown below.

The analysis proposed by Jones (1984, Figures 1 and 2) does not add any further knowledge. It clearly does not show that one model or the other provides any better fit to the data. The prediction he makes from the polynomial model is post hoc and, in fact, arbitrary. The pattern of the results as drawn in Jones's Figures 1 and 2 are exactly the same for the quadratic and exponential representations. Just take the horizontal line (that passes through the y-ordinate of zero), shift it down to a value of -.15 (in both figures), and the same results and conclusions that were drawn from the polynomial model can

The author's mailing address is: Institute for Perception, TNO, Postbox 23, 3769 ZG, Soesterberg, The Netherlands. now be obtained from the exponential model. Thus, as far as the Keren (1983) data is concerned, the two models lead to identical conclusions and there is no reason why one model should be preferred to the other.

In a previous comment, Jones (1979) gave three reasons why the polynomial model was preferable to the exponential. In the following, I briefly comment on each of the three arguments.

The first argument put forward was that the polynomial model was more parsimonious, because it required estimation of only one parameter instead of the two required by the exponential model. Although parsimony by itself is undoubtedly a desirable property, it is by no means the only criterion for evaluating a model. A model with two interpretable parameters is superior to an uninterpretable single-parameter model. The exponential model can be psychologically interpreted: In the analysis of my own data (Keren, 1983, Table 2), for instance, it is shown that subjects are underestimating the exponent by a factor of $\beta$, but are aware that the function is not simply linear or multiplicative. They are trying to compensate for the insufficiently large exponent by introducing a multiplying factor, $\alpha$, which is always larger than 1 . Obviously, that "correction" is not sufficient. In addition, what is shown in Table 2 is that being introduced to high inflation rates results in a more realistic (yet still not large enough) value for $\beta$. It is difficult to see what interpretation can be given to the different parameters of the polynomial model; Jones, at least, does not provide any.

In the polynomial model, the number of parameters required depends on the polynomial used. Jones (1979, p. 233) provides a table in which the order of the polynomial (used as the model) is determined by the gradient of the initial series. Obviously, some of the polynomials in this table require more than one parameter. As a matter of fact, the polynomial that Jones has fitted to my own data (Keren, 1983) contains three parameters, so that, according to the criterion offered by Jones himself, the exponential model should be preferred (since it is more parsimonious).

More disturbing, however, is the lack of any experimental support for the dependencies between polynomial extrapolation and gradient of initial series as proposed in this table. It is in this sense that I argued earlier that more empirical work was needed before a verdict could be made in favor of the polynomial model.

Jones's second argument was that "it is plausible that subjects should process the initial series relative to the analytically simplest form of accelerative function, the quadratic, rather than relative to the exponential function" (p. 233). This statement is again purely speculative and up to now has no empirical support.

Jones's last argument was that "the polynomial model has the advantage that it may also be applied to extrapolation from a wide range of series which are distributed 
other than exponentially"' (p. 233). First, this claim does not invalidate the exponential model in any way. Second, as already argued, such a generalization should be demonstrated empirically. In fact, following Jones's line of reasoning (i.e., speculating), my prior belief is that the relationships between magnitude of gradient and the order of polynomial proposed by Jones (1979) in Table 2 will not stand an empirical test. Jones is invited to challenge my belief.

\section{REFERENCES}

JoNES, G. V. (1977). Polynomial perception of exponential growth. Perception \& Psychophysics, 21, 197-198.
JONES, G. V. (1979). A generalized polynomial model for perception of exponential series. Perception \& Psychophysics, 25, 232-234.

JoNes, G. V. (1984). Perception of inflation: Polynomial not exponential. Perception \& Psychophysics, 36, 485-487.

KeREN, G. (1983). Cultural differences in the misperception of exponential growth. Perception \& Psychophysics, 34, 289-293.

WagenaAR, W. A., \& Sagaria, S. (1975). Misperception of exponential growth. Perception \& Psychophysics, 18, 416-422.

WagenaAr, W. A., \& Timmers, H. (1978). Extrapolation of exponential time series is not enhanced by having more data points. Perception \& Psychophysics, 24, 182-184.

WagenaAR, W. A., \& Timmers, H. (1979). The pond-and-duckweed problem: Three experiments on the misperception of exponential growth. Acta Psychologica, 43, 239-251.

(Manuscript received June 15, 1984; accepted for publication June 25, 1984.) 